\title{
The evolution of music
}

\section{In the second of a nine-part essay series, Josh McDermott explores the origins of the human urge to make and hear music.}

We think we understand why we are driven to eat, drink, have sex, talk and so forth, based on the uncontroversial adaptive functions of these urges. The drive to engage in music, a compulsion that is arguably just as pervasive in our species, has no such ready explanation. Music was one human behaviour that Charles Darwin was uncertain he could explain, writing in The Descent of Man, and Selection in Relation to Sex: "As neither the enjoyment nor the capacity of producing musical notes are faculties of the least use to man ... they must be ranked amongst the most mysterious with which he is endowed."

Music's origins have remained puzzling in the years since, although there is no shortage of speculation on the subject. Some argue that music is merely a side effect of traits that evolved for other functions. Our perceptual and cognitive abilities may have accidentally resulted in a system that finds pleasure and interest in musical stimulation. This idea should perhaps be the null hypothesis, and is by no means implausible. Music's perceptual basis could derive from general-purpose auditory mechanisms, its syntactic components could be co-opted from language, and its effect on our emotions could be driven by the acoustic similarity of music to other sounds of greater biological relevance, such as speech or animal vocalizations.

\section{Just-So stories}

Others contend that music is an adaptation with an associated biological function. Some (including Darwin) have proposed that music is the product of sexual selection. Alternative hypotheses include that music promoted social cohesion in group activities such as war or religion, that music was an evolutionary antecedent to language, or that its evolution was driven by the pacifying effect it has on infants.

These discussions run the risk of being mostly 'Just-So' stories, as there are few data with which to test or constrain theories. The fossil record provides scant clues, and many of the experiments that one might conduct to ask questions about adaptations in other species, such as removal of a trait to test the effect on fitness, are impractical or unethical. Moreover, any current effect on fitness, or lack thereof,

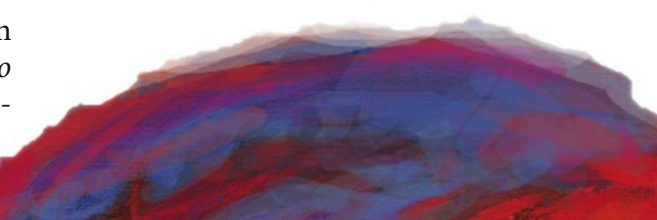

are shared with other cognitive domains. Despite not explicitly testing particular adaptationist theories, these two research directions nonetheless constrain theories of music's evolution in useful ways. Any music-related behaviour that has been selected for must have a genetic basis; and many aspects of music may not be so determined. A trait selected for its role in music might additionally be expected

to function specifically in music, and not in other domains. If some musicrelated trait - say, the ability to perceive pitch changes - were shown to overlap functionally with something that had a clear adaptive function, such as the perception of speech intonation, it would seem more likely that its role in music is a fortuitous side effect. No single such example is definitive, but repeated demonstrations of nonspecificity would render adaptationist hypotheses less plausible.

\section{Culture club}

The mere presence of music in every known culture implies some genetic basis. But music varies dramatically from culture to culture, and many aspects of musical behaviour seem at best only weakly constrained

by genetics. Whereas our ability to hear pitch intervals, for instance, could well be biologically rooted in the hardware of the auditory system, our emotional response to particular scales or chords seems likely to be acquired from exposure to a particular culture. Interactions between genes and environment are complex, and unravelling their contributions is not easy, but studies of music in different cultures and of musical development offer some hope.

A number of interesting music-related traits emerge in targeting two more modest goals. Empirical studies can help to identify musical traits that have a genetic basis, and can test whether these traits in human infants with fairly minimal musical input, providing some evidence for innate constraints. Babies notice when the notes of a 
melody are reordered, but not when they are shifted to a different pitch range. Infants, like adults, are sensitive to the relationships between notes, which is preserved in transposition, but altered by reordering. Infants also tend to be captivated by music relative to many other stimuli. Not all music is equivalent to them - they prefer combinations of notes that are judged by adults to sound pleasing, or consonant (the perfect fifth, for instance), over combinations that are less pleasing, or dissonant (a minor second). Infants may even extract metre from music: they react when the rhythm changes from a march to a waltz.

\section{Universal appeal}

Features of music that occur repeatedly around the world despite the substantial cultural variation in music also provide clues to genetically constrained mechanisms. Lullabies seem to qualify as a rare universal - nearly every culture has a genre of music geared towards infants, and there is considerable consistency in how they sound, generally being slow, repetitive and featuring descending pitch contours. Other features that are common, if not completely universal, among cultures include the inclination to dance to music, musical metre, and the hierarchical organization of pitch, giving structural prominence to particular notes over others.

Might any of these potentially heritable traits have evolved specifically for music? Examining functional overlap with other domains can provide insight. Language is generally the most popular candidate; both faculties combine discrete elements (notes/phonemes) into complex structures according to rules. There is growing evidence that this resemblance is more than superficial. Neuroimaging shows frequent overlap between the brain regions that language and music activate. Recent studies suggest that Broca's area - thought to be responsible for linguistic syntax - is also activated by chords that are inappropriate to their musical con-

"Music is a significant
feature of every known
culture, despite not
serving an obvious
adaptive function."

speech or other biologically relevant signals. Experiments manipulating this similarity could help to reveal whether there is an effect that is specific to music.

\section{Animals and music} Music perception in animals can also help to
determine the functional specificity of musical traits. Animals lack music; although some birds, whales and other species are said to 'sing', song is absent in most primates, including the apes, and as such is almost surely not homologous to human music. Any music-related trait found in animals, such as text, indicating that the area may have a role in musical 'grammar. Behavioural research also suggests that music and language comprehension can interfere with each other.

It is also conceivable, although largely untested, that much of the interest that young infants have in music is due to its similarity to a preference for some sounds over others, or a tendency to hear pitches an octave apart as similar, is thus likely to represent a more general-purpose mechanism. Similarities between human and nonhuman animals can thus indicate that a trait did not evolve for music.

Most studies on music perception in animals have emphasized their differences with humans rather than potential homologies. Animals, unlike adult and infant humans, have difficulty recognizing melodies that have been shifted up or down in pitch, for instance. Comparative data are generally more powerful when similarities are present across species rather than differences and, so far, few are evident for music. Many musical capacities, however, remain unexplored. Rhythm and metre, for instance, have been neglected, and could plausibly result from general-purpose mechanisms that some animals might share. The list of species that have been tested is also short; notably absent are our closest relatives, the apes, whose auditory systems and cognitive abilities are likely to be closest to our own.

\section{Enduring puzzle}

So far, it seems as if there are several aspects of musicality that emerge early in life with minimal exposure to music, and several that are present across diverse musical cultures. These traits may thus be partially heritable. It remains possible that none of these traits is specific to music. This would argue against the idea that music is an adaptation. However, there is much we still do not know, and future experiments could certainly alter this picture.

Music is universal, a significant feature of every known culture, and yet does not serve an obvious, uncontroversial function. As such it stands in contrast to other universal human behaviours. Speculation about its possible adaptive functions has been popular since the time of Darwin, and shows few signs of resolution. Empirical approaches offer a promising alternative. There is no guarantee that a full account of music's origins will ever emerge; in fact, that seems quite unlikely at present. Nonetheless, the right experiments will reveal a great deal - about the innate core of musical behaviour, the traits that might be unique to music, and the possible origins of those components that are not. All of which promises to enrich our appreciation of this human obsession. Josh McDermott is a postdoctoral associate studying hearing and music perception in the Department of Psychology, University of Minnesota, 75 East River Road, Minneapolis, Minnesota 55455, USA. 